\title{
Inviting a friend to evaluate potential grade III pancreatic injuries: Are they truly occult, or simply missed on CT?
}

\author{
Chad G. Ball, MD, MSc \\ Thomas S. Clements, MD \\ Andrew W. Kirkpatrick, MD \\ Kelly Vogt, MD \\ Walter Biffl, MD \\ Morad Hameed, MD
}

Accepted May 25, 2021

\section{Correspondence to: \\ C.G. Ball \\ 1403-29 St NW \\ Foothills Medical Centre \\ Calgary AB T2N 5A1 \\ ball.chad@gmail.com}

Cite as: Can J Surg 2021 December 21; 64(6). doi: 10.1503/cjs.001421

\section{SUMMARY}

Traumatic inuries to the pancreas are notoriously challenging to diagnose and treat. Detecting a main pancreatic ductal injury can be particularly difficult on screening computed tomography (CT). Twenty-four blinded faculty clinicians from 4 differing specialties and 6 institutions reviewed 9 video CT cases of potential pancreatic ductal injuries. Clinician performance in detection of confirmed grade III pancreatic injuries varied widely among specialties. This heterogeneity confirms the critical need for multidisciplinary care and image interpretation for even "minor" (i.e., not grade IV or V) potential pancreatic injuries to optimize outcomes for injured patients. The ubiquitous availability of electronic devices allows real-time collegial second opinions to be easily available.

$\mathbf{T}$ rauma to the pancreas is often deadly and always challenging. These injuries will test a surgeon's skills and demand great teamwork among surgical and nonsurgical colleagues. Given that computed tomography (CT) remains an insensitive test for detecting grade III pancreatic injuries (i.e., main pancreatic ductal damage),,$^{1,2}$ the primary goal of this audit was to evaluate the ability of faculty clinicians from varying backgrounds to identify grade III pancreatic injuries on blinded review of trauma-related CT imaging of the torso.

The provincial trauma registry was used to select 9 cases from a 5-year period of patients with American Association for the Surgery of Trauma (ASST) grade I $(n=2)$, II $(n=1)$ and III $(n=6)$ pancreatic injuries. Imaging for all patients was completed on a 256-slice GE scanner. Out of 216 individual case image reviews, the raters described the imaging as "adequate for diagnostic purposes" in $98 \%$ of cases. These Digital Imaging and Communications in Medicine (DICOM)-quality video torso CT files were evaluated by faculty radiologists $(n=6$, median years of experience [YOE] 17 , all academic trauma centres), trauma surgeons $(n=6$, median YOE 16 , all academic trauma centres), general surgeons $(n=6$, median YOE 19, 2 community and 4 academic centres), and high-volume pancreatic surgeons ( $n=6$, median YOE 20, all academic centres) from 6 institutions in 2 countries. Case details were not provided to the reviewers to ensure blinding and to reinforce focus on the pancreatic injury itself. Each patient underwent exploration with intraoperative confirmation of the pancreas injury grade (not necessarily as the primary indication, however). Confirmation of a grade III injury was obtained by a complete alignment of the final pathology report and intraoperative surgeon evaluation in all cases (as well as intraoperative pancreas ultrasound in 3 patients). Of these 9 patients, more than half $(56 \%)$ were female, the median age was 33 years, more than two-thirds (67\%) had a blunt mechanism of injury, the median Injury Severity Score (ISS) was 25, and most (89\%) were typically hemodynamically stable. Of the 6 patients with grade III injuries, $67 \%$ underwent an immediate $(<2 \mathrm{~h}$ after arrival) laparotomy; the remaining patients underwent a delayed laparotomy (3-6 h after arrival). All patients 
Table 1. Cohen $\kappa$ values for interobserver agreement across pancreatic injury cases

\begin{tabular}{|lcccc|} 
& \multicolumn{4}{c|}{$\kappa(95 \% \mathrm{Cl})$} \\
\cline { 2 - 5 } Specialist & \multicolumn{3}{c|}{ All cases $(n=9)$} & \multicolumn{1}{c|}{ Grade III cases $(n=6)$} \\
\hline General surgeons & 0.43 & $(0.22-0.80)$ & 0.29 & $(0.09-0.42)$ \\
\hline Trauma surgeons & 0.54 & $(0.19-0.74)$ & 0.33 & $(0.12-0.75)$ \\
\hline Radiologists & 0.82 & $(0.66-0.91)$ & 0.84 & $0.70-0.89)$ \\
\hline Pancreatic surgeon & 0.89 & $(0.78-0.94)$ & & 1.00 (Ref.) \\
\hline $\mathrm{Cl}=$ confidence interval; Ref. $=$ reference category. & & \\
\hline
\end{tabular}

with grade III injuries underwent resection (distal pancreatectomy, $83 \%$ with concurrent splenectomy) for body/tail injuries. One patient required damage control surgery. Most patients (83\%) with grade III pancreas injuries underwent concurrent intraoperative procedures, including colonic $(50 \%)$ and small bowel $(33 \%)$ repairs, as well as pelvic packing (17\%), lower extremity amputation (17\%), and craniectomy (17\%). Most patients $(83 \%)$ received a closed-suction surgical drain following a stapled $(67 \%)$ pancreatic transection $(50 \%$ added ductal suturing). The median length of stay was 11 (hospital) and 1 (intensive care unit) days. Although no patient died, $33.3 \%$ required insertion of a postoperative percutaneous drain for a pancreatic fistula/leak.

These 9 specific cases were carefully selected by our group to ensure a typical and accurate representation of patients with grade III pancreatic injuries, to avoid any concurrent upper abdominal injuries that could potentially influence or deter focus on the pancreatic injury itself, and to divorce the rater's diagnostic performance from individual case details. This methodology has been used previously by our group for both occult pneumothoraces and overt hemothoraces.

Clinical care for pancreatic diseases, whether benign (e.g., pancreatitis), malignant (cancer) or traumatic, has become one of the best examples within surgery of the need for advanced multidisciplinary care. Contributions from interventional radiologists, pancreatic surgeons, interventional gastroenterologists, acute care surgeons, critical care physicians, pancreas-specific radiologists, and even medical and radiation oncologists, have become essential to the delivery of comprehensive pancreatic care. From the initial diagnosis, to clinical therapy, to treatment of postoperative complications, this paradigm is equally important within pancreatic injury care. More specifically, detection of grade III pancreatic injuries (i.e., disruption of the main pancreatic duct) remains extremely challenging. ${ }^{1,2}$ Initial screening for nearly all severely injured patients is a CT scan of the torso with intravenous contrast. Unfortunately, this test has been shown repeatedly to be inaccurate in detecting pancreatic ductal injuries. ${ }^{1,2}$ Despite the debated usefulness and sequencing of subsequent investigations (magnetic resonance cholangiopancreatography, endoscopic retrograde cholangiopancreatography, endoscopic ultrasonography) as well as the tremendous utility of intraoperative ultrasonography performed by experienced pancreatic surgeons, ${ }^{1}$ potential differences in interpretation of initial trauma screening CT imaging remain unstudied. Variance in reporting of both radiologic studies and potential resectability ${ }^{3}$ have been previously described in detail. Similar to the preceding investigation of occult pneumothoraces,${ }^{4}$ the direct question remains: Is the suboptimal test performance of the investigation a result of a truly poor screening test, or is it a consequence of heterogeneity across interpreting physicians as a result of differing training and volume exposures?

To answer this question, each of the 24 clinicians involved in the review possessed the same video studies and no clinical details. Interestingly, but perhaps predictably, significant variance was identified across their interpretation of the CT studies (Table 1). Among the 9 cases reviewed, the mean correct interpretation of ductal (v. nonductal) injuries occurred among $44 \%$ of general (nontrauma) surgeons, $56 \%$ of trauma surgeons, $83 \%$ of radiologists, and $89 \%$ of high-volume pancreatic surgeons. When limited to the 6 patients with grade III injuries, success was $50 \%$ among both general and trauma surgeons, $83 \%$ for radiologists and $100 \%$ for pancreatic surgeons. Interestingly, with more challenging injuries, significantly increased heterogeneity was noted among both general and trauma surgeons (Table 1). In anecdotal conversation with many of the high performers, correct interpretation in these scenarios shifted to reliance on indirect signs of potential ductal injuries (e.g., pattern of parenchymal damage, location of peripancreatic fluid/ inflammation, knowledge of typical main duct location within the morphology of a given pancreas).

The primary message from these data is not that some clinician groups are superior to others in regards to diagnostic accuracy, but more importantly that collaboration with high-volume colleagues experienced in the diagnosis and clinical care of complex medical-surgical issues remains critical to the ultimate success of the patient. Given the realtime ability to share video files over numerous devices (e.g., smart phones), irrespective of time of day or night, the opportunity to collaborate with colleagues has never been more readily available. While there have been superb reports of this type of multidisciplinary operative care between trauma and pancreatic surgeons at some of our busiest international trauma centres, ${ }^{5}$ much less has been written regarding obtaining real-time colleague expertise within the initial diagnostic arena. The varying performance of surgeons and radiologists across disciplines shown in this report reinforce the need to involve our colleagues for diagnostic assistance when cases are less than straightforward. As with bile duct injuries, detection of pancreatic ductal injuries can be challenging; patients will benefit from high-volume subspecialty expertise. Unlike with bile duct 
injury repairs, however, transfer to a high-volume pancreatic surgeon's care may not be required if the general or trauma surgeon has adequate expertise to perform a distal pancreatectomy and splenectomy. Managing complications related to the pancreas, such as leaks, would also be a mandatory skill.

\section{Conclusion}

The role of multidisciplinary surgical care for injuries to the pancreas remains critical to achieving optimal outcomes for severely injured patients. Given the current availability and capacity of our electronic devices to share video CT files, obtaining real-time second opinions regarding both the diagnosis and care of patients with potential pancreatic injuries has never been easier. If it's about the patient, then we should all be willing to invite a friend.

Affiliations: From the Department of Surgery, University of Calgary, Calgary, Alta., Canada (Ball, Clements, Kirkpatrick); the Department of Surgery, Western University, London, Ont., Canada (Vogt); the Scripps Memorial Hospital, La Jolla, California, USA (Biff); and the Department of Surgery, University of British Columbia, Vancouver, BC, Canada (Hameed).

Competing interests: C.G. Ball is co-editor-in-chief of CJS; he was not involved in the review or decision to accept this paper for publication. A.W. Kirkpatrick has consulted for Zoll, Acelity (3M/KCI), CSL Behring, Innovative Trauma Care and SAM Medical Corporations, and he is the principal investigator of a randomized trial partially supported by Acelity. He has also received equipment from Zoll, SAM Medical Corporation and Innovative Trauma Care. No other competing interests were declared.

Contributors: All authors contributed substantially to the conception, writing and revision of this article and approved the final version for publication.

Content licence: This is an Open Access article distributed in accordance with the terms of the Creative Commons Attribution (CC BY-NC-ND 4.0) licence, which permits use, distribution and reproduction in any medium, provided that the original publication is properly cited, the use is noncommercial (i.e., research or educational use), and no modifications or adaptations are made. See: https://creativecommons.org/licenses/by-nc-nd/4.0/

\section{References}

1. Streith L, Silverberg J, Kirkpatrick AW, et al. Optimal treatments for hepat-pancreato-biliary trauma in severely injured patients: a narrative scoping review. Can 7 Surg 2020;63:E431-4.

2. Byrge N, Heibrun M, Winkler N, et al. An AAST-MITC analysis of pancreatic trauma: Staple or sew? Resect or drain? 7 Trauma Acute Care Surg 2018;85:435-43.

3. Aubin JM, Bressan AK, Grondin S, et al. Assessing resectability of colorectal liver metastases: How do different subspecialties interpret the same data? Can 7 Surg 2018;61:251-6.

4. Ball CG, Kirkaptrick AW, Feliciano DV. The occult pneumothorax: what have we learned? Can F Surg 2009;52:E173-9.

5. Krige J, Nicol A, Navsaria P. Managing grade 5 pancreatic injuries think smart, act smart and call in the pancreatic cavalry early. 7 Trauma Acute Care Surg 2017;82:1187-8. 